\title{
EduCACIÓn DEMOCRÁtiCa, UN DESAFío
}

\author{
Democratic Education, a Challenge
}

Ph.D. Edesmin Wilfrido Palacios Paredes wpalacios@uisrael.edu.ec Universidad Israel - Ecuador

Fecha de recepción: 04/01/2015

Fecha de aceptación: 27/01/2015

\section{Resumen}

La educación de por sí es democrática, pues parte del principio de relacionar, educar, extraer, formar e instruir. Sin embargo, los principios no siempre son evidenciados y practicados.

El presente artículo tiene como objetivos fundamentales poner en evidencia la relación conceptual de algunos teóricos, que a través de la historia, han cuestionado la educación por ser unidireccional y conductual. Por otro lado, se pretende proponer algunas alternativas de interpretación sobre la educación democrática, con el firme cuestionamiento de que una educación sin democracia no es educación. La propuesta es que a mayor libertad, mayor responsabilidad y a mayor educación, mayor democracia.

Palabras clave: Democracia, educación, principios, cuestionamiento, libertad.

\section{Abstract}


Education per se is democratic, because of the principle of relating, educate, extract, training and instruction. However, the principles are not always practiced and evidenced.

The main purpose of this article is to demonstrate the conceptual relationship of some theorists who through history have questioned the education as unidirectional and behavioral. On the other hand, is focus on to propose some alternative interpretations about the significance of "democratic education", strongly questioning that an education without democracy is not an education. The proposal is that greater freedom, greater responsibility and at higher education, greater democracy.

Keywords: Democracy, education, principles, questioning, freedom. 


\section{Introducción}

La educación es tan elemental para el hombre en la sociedad, como es el agua para el cultivo de las plantas. Así como dice (Juncosa, 1990), la poda endereza la planta y revitaliza en función de producir frutos en abundancia, así la educación instruye al hombre y mejora sus comportamientos, sus acciones son más coherentes, razonables y astutas; porque enseña y transforma (Prien, 1978), (López, 2004). Transforma desde la perspectiva de la educación ancestral, es decir, la educación inicial la que nuestros padres nos enseñan. Desde la concepción amerindia, (Reascos, 2000) menciona que la educación de nuestra madre es la que transforma y libera, porque nos enseñan a partir de las acciones, ejemplos, testimonios y sobre todo desde las alternativas vivenciales. La educación nos ayuda a los seres humanos a ser parte fundamental del mundo en el que vivimos, en el ámbito relacional, existencial y desde las prácticas familiares cotidianas (Rodríguez, 1987).

Para adentrarnos más en el tema y profundizar acerca de la educación, analizaremos desde algunos aspectos: los conceptos básicos, el significado y la importancia de la educación para el desarrollo de los pueblos. (Macas, 2005) Proponer elementos de formación teórica en el ámbito democrático, educativo y pedagógico, que le permita a la comunidad educativa y sociedad en general encontrar nuevas alternativas de convivir cotidiano, para el bien común.

\section{Educación democrática}

Desde los comienzos de la era humana, la educación ha dado la pauta para el desarrollo de la ciencia, la tecnología, el mejoramiento para el convivir cotidiano, y por eso se le atribuye el carácter de enseñanza, aprendizaje, crianza y conducción; elementos que son esenciales para el desarrollo social. Todos los seres vivos estamos destinados a nacer, crecer, aprender, practicar y ejecutar; y al mismo tiempo, a dejarnos enseñar por otros, eso es precisamente lo que analizaremos. La enseñanza de los otros, ¿Hasta qué punto nos ha beneficiado? ¿Nos ha instruido? y ¿Nos ha educado para vivir en la sociedad? (Erasmo, 1991); (Salomón, 1976).

Rousseau define a la educación de la siguiente manera: "Educación es igual a alimento Educit obstetrix, dice Varrón: educat nutris, instituit paedagogus, docet magister. Educación institución e instrucción, son por tanto tres cosas tan distintas en su objeto, como nodriza, ayo y maestro" (Rousseau, 1979, pág. 35). 
La educación es tan antigua como la humanidad, pues el hombre siempre se preocupó de "criar" y "enseñar" a sus hijos. En esta acepción inicial puede verse ya la raíz etimológica del concepto de educación, que procede del término latino educare, cuyo significado es "criar", "alimentar" o "instruir" (Rousseau, 1979).

No existe nadie en este mundo que nazca con todo su potencial desarrollado, todos necesitamos de un proceso, apoyados por la naturaleza y por el espíritu que fortalece el alma y potencializa las acciones en bien o en mal, dependiendo de la formación que haya recibido y de los intereses que tenga el hombre para consigo mismo y sus iguales (Rousseau, 1969).

Ahora bien, la educación es dirigir, encaminar y tiene una acción docente que conduce, guía, doctrina, controla; pero también es desarrollar y perfeccionar las facultades intelectuales, éticas y morales del niño/niña, joven o adulto (Montes, 1989). En este punto se inicia el primer problema que nace a partir del término educar o enseñar: que significa criar, alimentar, encaminar, conducir de adentro hacia afuera, extraer. Esto anuncia ya una fuerte carga idealista, incluso hasta esencialista, ya que éstos términos y éstas prácticas se arrastran hasta nuestros días; se trata de lo que había mencionado anteriormente. La educación es criar, es como el abono para la agricultura o la poda para la estabilidad y fertilidad de la planta.

Aquí se desprende la concepción mayéutica de la educación, mientras más libre de obstáculos exteriores, mucho mejor para el desarrollo del niño/niña, y sobre todo para que potencialice su capacidad intelectual, sus actitudes y aptitudes.

Durkheim, definió la educación como "la acción ejercida por las generaciones adultas sobre aquellas que no han alcanzado todavía el grado de madurez necesario para la vida social" (Durkheim, 1979). Con esto clarificamos más el problema que la educación, como enseñanza, pues la educación tiene por objeto suscitar y desarrollar un cierto número de estados físicos, intelectuales y morales que exigen del niño y niña, tanto la sociedad política como el medio ambiente específico al que está especialmente destinado.

Esto nos permite aclarar más el problema de la educación: no se trata de educar, criar, enseñar; sino de socializar, no tiene que ir de afuera hacia adentro, sino de forma viceversa de (Sousa, 2007) y no se trata de introducir los conocimientos al niño o niña, sino de potencializar y ayudar que el niño o niña aflore y descubra sus conocimientos.

La educación no es memorística, ni discursiva, sino que es experiencial basada en el contexto (Cordeiro, 2002).

Otro de los problemas o condiciones de la educación, es que el niño o niña está limitado/limitada a la personalidad y método de enseñanza del educador, el alumno se convierte en un mero seguidor y cultivador de la metodología de su maestro, empieza a buscar paradigmas en otros y no a descubrir su propio paradigma, su propia metodología de aprendizaje y enseñanza, el niño o niña queda limitado a los que los otros pueden dar y no a lo que él mismo puede dar de su potencial intelectual. (Pelauer, 2009), en un tercer momento menciona que el niño está limitado o destinado a ser inducido a una sociedad que los demás construyeron, y que los adultos quieren que sea como ha sido en el pasado. 
Con esta idea, la educación pretende educar y formar a la niñez y juventud, pero no tiene en cuenta la realidad del niño o de la niña, su individualidad, su conocimiento, que pueden aportar a la sociedad, elementos nuevos de comportamiento y conocimientos innovadores que mejoren la sociedad y las tradiciones del pasado, limitando la prosperidad, la creatividad y las posibilidades de nuevos paradigmas.

Todos y cada uno de nosotros recibimos formación de nuestros maestros, sin embargo eso no es todo, porque hay cosas que aprendemos por nuestra reflexión y desde nuestra propia experiencia. Además somos seres adaptados a aprender de los expertos a los que pertenecemos. (Mijia, 1988).

La historia confirma y los hechos ratifican que cada cultura confirió a la educación el enfoque que imponían las diferentes concepciones filosóficas, políticas y religiosas. Así cabe considerar que el proceso de enseñanza, consiste en la transmisión de valores y conocimientos de una determinada cultura. (A., 1994).

La educación entonces, es un proceso intencional que pretende al perfeccionamiento del individuo como persona y la inserción de éste, en el mundo cultural y social, entendiendo al proceso educativo como una parte activa en las sucesivas etapas de su larga y nunca concluida formación como individuo y como ser social (Reale, 1991)

Haciendo un ligero proceso evolutivo de la vida del ser humano, se descubre que el hombre es un ser social desde que está en potencia para ser engendrado. El embrión, al depender de la madre, se alimenta y se deja criar, por ello, al nacer, inmediatamente está en relación con los otros, con el medio que le rodea, aprende a vivir y es instruido. Por eso el proceso es interminable y se define como intencional, en cuanto que la educación se presenta como una acción planeada y sistematizada que tiende a un fin (Martinez, 1996).

Al individuo se le considera libre para actuar y ejecutar su creatividad sin determinismos previos, además alude al perfeccionamiento del individuo como persona, porque el proceso educativo entraña una modificación y desarrollo de todas las posibilidades del ser humano, primordialmente en sus aspectos más específicos como: inteligencia, voluntad, plano físico y sensorial, a fin de conseguir el desarrollo integral de la persona. (Erasmo, 1991).

Por consiguiente la educación influye en la totalidad de la persona porque se distingue de la mera instrucción y formación: entendida la instrucción como la fase de la enseñanza y el aprendizaje que afecta sobre todo a la inteligencia y a la adquisición de conocimientos. Y la formación hace referencia la instrucción ya asimilada e integrada por el individuo en algún aspecto de la persona. Sin embargo la educación atañe a todas la etapas, facetas de la personalidad global que exige un proceso permanente de perfeccionamiento aunque jamás alcance la perfección total (Maturana \& Varela, 2001).

Todos estamos inmiscuidos en este proceso de aprender y dejarse criar por los demás, porque somos necesarios en este proceso de enseñanza - aprendizaje, por nuestra originalidad, por la idiosincrasia individual, es decir, por la identidad propia de cada uno, donde se hace útil para el otro y se enriquece mutuamente; por lo tanto es recíproco el objeto de la educación (Rana R. H., 1998). 
A través de la historia, en diferentes culturas y periodos, el hombre ha desarrollado diferentes modelos educativos. En distintas civilizaciones, como la egipcia, babilónica, persa, romana. "La educación estaba supeditada a los intereses de la religión y del sistema teocrático del gobierno y de las tradiciones" (erasmo, 1991). Por consiguiente, es importante analizar el espíritu, el material y los métodos de la educación a partir de los diferentes tipos de vida de la comunidad.

Admitir que la educación enseña, instruye a los seres ignorantes o inmaduros mediante el proceso correspondiente de enseñanza durante el periódo de educación básica superior, equivale a decir que la educación varía con la calidad de vida del grupo al que pertenece el individuo. En este sentido, es verdad que una sociedad que no sólo cambia sino que tiene el ideal de tal cambio, posee normas y métodos de educación diferentes de aquella sociedad que aspira solamente a la perpetuación de sus propias costumbres. (Dewey, 1952).

\section{La educación como ideal democrático}

En un primer momento se considera que el desarrollo y la tranquilidad armónica de la sociedad dependen de una buena formación y buena utilización del material potencial intelectual humano. En un segundo momento es fundamental un cambio de hábitos sociales, una interacción más libre entre los grupos sociales, que deberán formar parte de una sociedad más organizada y con un ideal democrático en el pensar y en el actuar, partiendo del principio indispensable que es el derecho a la vida y el respeto del otro como ser humano (B., 2007).

Desde un punto de vista educativo se hace necesaria una sociedad democrática más interesada en organizar una educación pensada y sistemática. Por medio de la educación se puede alcanzar un equilibrio y una armonía social. Por tanto lo que hace que mejore una sociedad democráticamente construida es el desarrollo de una mayor interrelación personal, la ampliación de los intereses compartidos, la liberación de una mayor diversidad de capacidades intelectuales (Gutmann, 1987).

Una sociedad desorganizada establece un gran número de modelos y normas diferentes, y en tales condiciones, es imposible para el individuo alcanzar una consistencia espiritual y moral. Esto último sólo es posible en un todo completo y armonioso. Una sociedad que se apoya en modelos confusos e independientes, se divide en la abstracción y antagonismo, con lo cual es imposible que se logre una armonía en la construcción de una sociedad democrática (Dewey, 1952). En estas condiciones, la educación no cumple con su papel de organizar y orientar. Sólo un estado justo justo será capaz de proporcionar la educación debida.

Ahora bien, las posibilidades de llegar a una educación debida resultan escasas, debido a la dificultad de construir una sociedad democrática. Podemos apelar a Platón para hallar una salida a esta situación que se asemeja a un callejón sin salida. Según Platón, los hombres filósofos amantes de la sabiduría pueden aprender por el estudio y llegar a la verdad, pueden aprender y descubrir modelos de existencia verdadera (Reale, 1991). Si un gobernante, dentro de sus políticas de estado, retomara modelos de sabiduría y por medio de la educación realizara proyectos que estén acorde a la realidad social, estos modelos podrían convertirse en reglas para la 
gobernabilidad, y podría aplicar una educación que permita a los individuos descubrir su naturaleza de ser y sus aptitudes, de esa forma pondrían en función de la sociedad sus capacidades y cualidades participando de una manera real, eficiente y consciente (Morales, 2007).

Por tanto, se puede afirmar que desde el pensamiento filosófico y pedagógico, lo fundamental para la construcción de una cultura democrática es en primer lugar, dar significado social al papel de la organizaciones sociales; y en segundo lugar, profundizar en la relación que el Estado, la Educación y el Ciudadano guardan (Hernández, 2007).

La educación debe ser el proceso que le ayuda al individuo a descubrir su propia naturaleza de ser desde su condición social, su situación política y económica. Una vez que el individuo descubre su naturaleza inmediatamente encuentra su razón de ser, de existir y actuar.

El proceso educativo le permite al individuo encontrar su labor en función de un principio esencial que es el de existir para ser feliz y realizado. Por tanto, la educación es el elemento que le facilita descubrir las capacidades, prepararlas y ponerlas al servicio del bien común, dejando de lado determinismos.

Si para Platón los individuos nacen para un fin, y ese fin es encontrar la perfección por medio del estudio y su naturaleza a partir de la educación, la sociedad a la que pertenece debe buscar en la preparación de los individuos, hombres políticos, reflexivos y dispuestos a vivir democráticamente.

El individuo aislado no es nada; sólo en y por la absorción de los objetivos y el sentido de las instituciones organizadas alcanza su verdadera personalidad (Dewey, 1952).

Kant define la educación, en su tratado de pedagogía, como el proceso por el cual el hombre llega a ser hombre; así como la educación permite que se llegue a la perfección. Pero para que una sociedad esté encaminada a la perfección, depende de otras cosas: de que los hombres se esfuercen por educar a sus sucesores, para que el ideal de sociedad sea una construcción generalizada y no particular, por las siguientes razones:

En primer lugar los niños, niñas y jóvenes son intelectualmente imaginativos, activos y creadores, por lo tanto sus aprendizajes no sólo son guiados por mecanismos adaptativos. El estudiante tiene la capacidad de innovar, razonar, discrepar, sentir y pensar, si tiene la oportunidad para ello.

Estas habilidades no surgen espontáneamente sino que son aprendidas en contacto directo con preguntas y retos, experiencias que los activan intelectual y emocionalmente. Sin lugar a dudas, un estudiante que sólo memoriza y repite las fórmulas que le da el docente, al cabo del tiempo no tendrá habilidad para plantearse dudas e inquietudes que movilicen sus capacidades intelectuales y emotivas (Jürgen, 2000).

El estudiante en este enfoque debe ser el protagonista de su propio proceso de conocer. No debe convertirse en un ser pasivo que se sienta a esperar que le digan lo que debe hacer o en ocasiones responder cuando el docente 
pregunta. El estudiante puede iniciar el proceso, traer problemas al aula o fuera de ella que se relacionen con el tópico a estudiar tener dudas y retos que con la ayuda del docente aprende a dilucidar.

Relacionado con lo anterior, está implícita la idea de que el objeto de la educación no es que los alumnos repliquen y comprueben las verdades conocidas de las cuales son informados: más bien es motivarlos a visualizar una realidad, una ciencia, una tecnología y conocimiento cultural que siempre se está haciendo, que siempre está en construcción a través de un aprendizaje experiencial.

Visto así, lo más importante es que el estudiante se sitúe frente a la experiencia de aprendizaje con una actitud y una conducta responsable e independiente. Que no haya que recurrir necesariamente a castigos, amenazas, calificaciones, notas, exámenes, reportes a la familia, para que aprenda.

Esto no significa que no haya evaluaciones, o que el estudiante no trabaja, sólo que lo hace como parte de un proceso necesario para apropiarse del conocimiento y no para obtener buenas notas o evadir las malas (Santos, 2002).

Lo anterior nos señala otro principio, que difiere, en algún sentido, de la posición tradicional que enfatiza la competencia individual y el logro personal. Para el aprendizaje experiencial la aprobación y construcción del conocimiento cultural, aunque es una responsabilidad y tarea personal, es a la vez un proceso social.

El aprendizaje no se da en forma aislada, o solos ante un libro o frente a un paquete programado. Lo que permite el crecimiento personal y el trabajo grupal, es la cooperación, el lidiar juntos con el problema, el aprender a escuchar argumentos, a ponerse en el lugar de otros, el compartir alternativas y buscar caminos (Lopes, 2004). Cuando la idea y los procedimientos que cada cual utiliza se confrontan con los de los demás en el interior de un proceso estructural, se produce el conocimiento.

En un espacio de interacción es posible negociar varias propuestas y evacuar la viabilidad de ellas. En esta idea de interacción subyace un objetivo fundamental: es, que el individuo realmente aprenda de la experiencia, que no pase por la información y el conocimiento de una manera superficial (Santos 2002).

A continuación, se describen dos formas de abordar el problema del aprendizaje (Tabla 1). Tal vez podemos reconocer algunas de las prácticas de los estudiantes, quienes lejos de construir el conocimiento son inducidos, más bien, a movilizarse por otro objetivos.

Por su puesto, el que un estudiante siga uno u otro camino depende de la forma en que el facilitador organiza la experiencia del aprendizaje del currículum oculto, del rol activo o pasivo que el estudiante cumple en el proceso de aprender, de lo significativo del material que debe aprender, de la metodología que se siga en el curso, de sí fomenta el logro individual y los estímulos como manera de asegurarse el rendimiento académico, o el proceso de interacción experiencial que provoca retos y activa la motivación para resolver conjuntamente los problemas (Laporta, 1985). 
Tabla 1: formas de abordar el problema del aprendizaje.

\begin{tabular}{|l|l|}
\hline \multicolumn{1}{|c|}{ Enfoque estratégico } & \multicolumn{1}{|c|}{ Enfoque experincial } \\
\hline $\begin{array}{l}\text { Intención de obtener notas lo más altas posible. } \\
\text { Uso de exámenes previos para predecir preguntas. } \\
\text { Atento a pistas sobre esquemas de puntuación. } \\
\begin{array}{l}\text { Organiza el tiempo y distribuye el esfuerzo para obtener } \\
\text { mejores resultados. }\end{array}\end{array}$ & $\begin{array}{l}\text { Se tiene en cuenta las necesidades de estudiante. } \\
\text { Intención de comprender procesos. } \\
\text { Fuerte interacción con el compromiso a partir de la expe- } \\
\text { rienciara meteriales adecuados y condiciones de estudio. } \\
\text { Relación de nuevas ideas con el conocimiento anterior. } \\
\text { Relación de conceptos con la experiencia cotidiana. }\end{array}$ \\
\hline
\end{tabular}

Fuente: autor.

Un aspecto que ha requerido mucho análisis en esta mirada de la educación es el referido a esquemas conceptuales, ideas, marcos de referencia y representaciones mentales que los alumnos traen a la experiencia de aprendizaje. Existe un reconocimiento que tanto los estudiantes como los profesores no aprenden como sujetos vacíos de sus ideas, su mente no es una tabla rasa o un papel en blanco sobre el cual el profesor escribe, tampoco un recipiente vacío que la escuela llena de conocimiento presumiblemente útil. Es importante que el estudiante intente desarrollar habilidades investigativas que le ayuden a actualizar su conocimiento y aprovechar la información que va apareciendo para dar respuesta a problemas concretos (Santos, 2002).

Para esto, son importantes los momentos experienciales (talleres, clases, exploraciones) en los que se materializan situaciones abiertas donde los sujetos se relacionan con el objeto a estudiar y crecen en el proceso, es un espacio de intercambio de experiencias, ideas e información; es lugar para pensar y sentir, enfrentándose a la realidad (Lopes, 2004). Es una experiencia que permite discrepar, en donde cada cual tiene tareas precisas que conducen a un desarrollo de todos. En las actividades a desarrollar existe un método a seguir, no es asunto de cada uno hacer lo que quiere sin ninguna organización o finalidad requiere de un planteamiento que les produzca emoción y al que le vean sentido.

Este enfoque experiencial-constructivista-democrático se debe desarrollar en una organización circular que se centre en problemas más que en materias. Se le ve la necesidad de integrar los contenidos y que las materias no se vean aisladas unas de otras. Se trata de organizar el material alrededor de temas o problemas fundamentales que faciliten su comprensión. Este es un aspecto que difiere en lo fundamental de la clásica organización del programa de curso por contenidos o del currículo por asignaturas (Gutmann, 1987).

La formación del estudiante, desde un enfoque experiencial-constructivista y democrático, no tiene como finalidad darle solamente contenidos culturales o científicos; tampoco pretende prepararlo para que se adapte a normas o patrones establecidos, sino más bien para que aprenda a construir su propia vida junto a otros dentro de un marco de respeto hacia la heterogeneidad y la diversidad de la vida humana. De ahí la necesidad de la interacción, del desarrollo grupal, de las relaciones interpersonales en el salón de clases, en el espacio escolar, 
en el espacio ciudadano y en el escenario natural. El aprendizaje es también un ejercicio en la convivencia social y en la solidaridad y no sólo en la competencia individual y el logro personal (Rockwell, 1996)

En la actual normativa educativa ecuatoriana, encontramos que sus fines orientan a la formación en el respeto de la vida y los derechos humanos, a los principios democráticos de convivencia, pluralismo, justicia, solidaridad y equidad. También en los objetivos comunes para todos los niveles de la educación formal encontraremos la intención explícita de fomentar en la institución educativa, prácticas democráticas para el aprendizaje de los principios y valores de participación ciudadana y estimular la autonomía y la responsabilidad (Cortina, 1993).

La propuesta aquí presentada sobre grupos de apoyo, no es más que una de las variadas posibilidades que se tiene para transformar el currículum oculto. Entre otras, podemos destacar la escuela de líderes, consejo estudiantil, utilización del observador del alumno, manual de convivencia, escuela de padres, comités de profesores de curso, etc.

A lo largo de la historia pedagógica orientada al trabajo de grupo, existen entre otros las experiencias y aportes de Makarenko, Don Bosco, Piaget, Vigotsky, Kohlberg. Estos autores enfatizan en las relaciones solidarias de los estudiantes, plasmadas en estructuras organizativas donde se transita de una escolarización competitiva e individualista a una concepción que se fundamente en la activa participación de los estudiantes (Thiebaut, 1994).

De una u otra forma, proponen que los estudiantes se organicen en equipos de trabajo, donde los conflictos sean los carburantes para desequilibrar continuamente su propio proceso formativo. Lo anterior es posible si lo que están haciendo lo estudiantes es significativo para ellos y el ambiente en el que se desenvuelven es el grupo pequeño, donde ideas, perspectivas, expectativas y opciones entran en una interacción que obliga a buscar nuevas alternativas; estas nuevas posibilidades impactan en los individuos, desequilibrando el estado cognitivo, emocional, comportamental anterior y nuevas construcciones se generan como síntesis parciales del desarrollo del grupo al cual pertenecen. Estos saltos cualitativos se generan en lo intelectual, en lo afectivo, en lo valorativo, lo que impulsa a estructurar nuevas formas manifiestas de conductas en las estudiantes (Hermann, 2002).

Piaget, Vigotsky y Kohlberg proporcionan algunos conceptos que posibilitan la comprensión del desarrollo de niños, niñas y jóvenes cuando nos indican que el conocimiento y, en general, la actividad mental se estructuran en la interrelación dialéctica de los individuos y que el proceso de desarrollo de los chicos y chicas consiste en interiorizar gradualmente con lo que con anterioridad se ha logrado con el apoyo de otros. De otra manera se puede decir que el niño o niña va progresando en su capacidad para realizar ciertas actividades por sí solo, luego hay cosas que no puede hacer ni solo ni con ayuda, más adelante consigue hacerlas con ayuda de alguien más experto y por último, llegará a realizarlas de forma independiente (Santos, 2002).

La diferencia entre lo que un niño o niña pueden hacer con la ayuda de otro más experto es lo que Vigotsky llamó zona de desarrollo próximo, donde en cada nivel de desarrollo los estudiantes deberán distinguirse en dos momentos: el que corresponde al desarrollo efectivo que ha alcanzado un niño (lo que puede hacer por sí solo) 
y luego el nivel desarrollo potencial, es decir aquello que está desarrollándose pero que aún no es dominado por el niño o niña (Thiebaut, 1994).

En los procesos educativos, cualquiera que sea el trabajo realizado, deberá ubicarse en la zona de desarrollo potencial, pues el estudiante entraría en contacto con destrezas, habilidades y conocimientos que se encuentran en un estado rudimentario, embrionario, que posibilita un trabajo de apertura de nuevos espacios, de nuevos conceptos y de nuevas prácticas (Santos, 2002).

La antítesis de los anterior la encontramos cuando el proceso se sitúa en la zona de desarrollo efectivo del estudiante: trabajar allí sólo germinará uno que otro conocimiento específico, pero estará muy distante para conseguir nuevas destrezas de pensamiento, autorregulaciones valorativas y de manejo de autocontrol comportamental, para esto es importante tener en cuenta lo que dice Jhon Dewey:

"La dirección de grupo centrada en el apoyo intenta ubicarse en el trabajo en grupo de iguales, donde las diferencias de los estudiantes jaloneen sus procesos y desencadenan en acercamientos al desarrollo potencial" (Thiebaut, 1994).

"La dirección de grupo centrada en el apoyo es la conformación de una verdadera red de células democráticas en todos los cursos de institución educativa. Debe ser una opción institucional y no sólo de algunas personas. Esta estructura educativa, es parte fundamental del Proyecto Educativo Institucional Democrático" (Dewey, 1952).

\section{Conclusiones}

La intención básica de la educación para la democracia es la formación integral de los estudiantes; de ahí la importancia que los directivos y docentes privilegian los espacios de formación en la estructura escolar. La dirección de trabajo en grupo, es uno de los espacios donde se concreta el desarrollo integral del estudiante como miembro de la comunidad educativa, y como futuro ciudadano de una nación donde se privilegia la participación y la justicia.

La justicia entendida como lo bueno, real, participativo y equitativo. Desde lo equitativo entendemos que la educación debe ser igual para todos en términos generales, es decir, el orden, el respeto y todos los valores universales. La educación ancestral comienza basada en alternativas, donde los seres humanos en la edad infantil reciben varios métodos de aprendizaje, en unos casos, una educación dialogante, relacional, entre la naturaleza y la sociedad.

La educación democrática, por lo tanto, es una educación vinculante y relacional complementaria, que necesitamos todos: niños, jóvenes y adultos. Porque la educación es y será siempre la forma más correcta de la emancipación de las ataduras conceptuales, ideológicas y culturales. 


\section{BIBLIOGRAFÍA}

A., M. R. Ancestralidad del pueblo Quichua. Abya-yala. Quito - Ecuador. 1994.

B., M. El estigma de los pueblos indígenas, su vida cotidiana. editorial Musa. Asunción - Paraguay. 2007.

Cordeiro, S. R. Vidas de artes educadoras. Editorial UFRGS. Porto Alegre. 2002.

Cortina, A. Ética aplicada y democracia radical. Editorial Tecnos. Madrid - España. 2002.

Dewey, J. Democracia y educación. Lozada. 1952.

Durkheim, E. La educación como socialización. Editorial Sígueme. Salamanca. 1979.

Erasmo, C. Diccionario Enciclopédico. E. U.: Enciclopedia Británica Publisher.

Gutmann, A. Educación y Democracia. Editorial Prisma. México. 1987.

Hermann, N. Hermenêutica e Educação. Editorial DP\&A. Rio de Janeiro - Brasil. 2002.

Hernández, E. El Estado y la interculturalidad en el Ecuador. Editorial ICONOS, 27. Ecuador. 2007.

J, R. J. Emilio o de la Educación. Editorial Porrúa. México. 1972.

Juncosa, J. Interculturalidad y a educación tradicional desafíos y experiencias. Editorial Abya-Yala. Quito. 1990.

Jürgen, H. Ideas y valores. Santafé de Bogotá. 2000.

Laporta, F. El principio de igualdad: introducción a su análisis en $<<$ Sistema $>>$. Madrid. 1985.

Lopes, D. Educación primaria y educación de tradiciones. Editorial Albuja. Quito. 2004.

Macas, L. Los pueblos y los diálogos entre culturas. CCES. Quito. 2005.

Martínez, M. Una propuesta pedagógica para educar en valores. Revista Pensamiento Educativo, 185-209. 1996.

Maturana. H. R. \& Varela, F. J. Árvore do conhecimento. Palas Athena. Brasil. 2001. 
Maturana. H. R. Emoções e linguagem na educação e na política. UFMG. Belo Horizonte. 1998.

Mijia, E. Formación y deformación construcción de identidades. Sarmiento. Quito. 1998.

Montes, A. Campos de educación trasformaciones sociales. Bautista. Quito. 1989.

Morales, F. El proceso plurinacional y las nacionalidades. SPPC. Quito. 2007.

Pelauer, D. Compreender Ricoeur. Vozes. Porto Alegre. 2009.

Prien, G. Caminos y desafíos en la educación ancestral. Editorial Abya-Yala. Quito. 1978.

Reale, G. y. Historia del pensamiento filosófico y científico. In J. A. Iglesias. Editorial Herder. Barcelona. 1991.

Reascos, M. Nociones de indio, runa, cultura. Editorial Andina. Quito. 2000.

Rockwell, V. Pensamiento indio y pensamiento blanco. SPPC. Quito. 1996.

Rodríguez, B. Creencia e identidad, cambio religioso y cambio cultural. Tierra Nueva XXV. México. 1987.

Rousseau, J. El contrato social, nuestros clásicos. UNAM. México. 1969.

Rousseau, J. Emilio o de la Educación. Editorial Bruguera. Barcelona. 1979.

Salomon, H. Costumbres y normas de la familia. Absec. Quito. 1976.

Santos, K. S. Os contornos da Escola: Organização Curricular por Ciclos, Espaços Escolares e Dificuldades de Aprendizagem. UFRGS. Porto Alegre. 2002.

Sousa, A. Educación superior en Brasil y Argentina: investigaciones antropológicas en Brasil y Argentina. Antropofagia. Buenos Aires. 2007

Thiebaut, C. El espacio político de las diferencias. Revista de libros. Fundación Caja de Madrid. Madrid - España. 1994. 
